\title{
A systematic review of modelling approaches in economic evaluations of health interventions for drug and alcohol problems
}

\author{
Van Phuong Hoang ${ }^{1 *}$, Marian Shanahan ${ }^{1}$, Nagesh Shukla ${ }^{2}$, Pascal Perez ${ }^{2}$, Michael Farrell ${ }^{3}$ and Alison Ritter ${ }^{1}$
}

\begin{abstract}
Background: The overarching goal of health policies is to maximize health and societal benefits. Economic evaluations can play a vital role in assessing whether or not such benefits occur. This paper reviews the application of modelling techniques in economic evaluations of drug and alcohol interventions with regard to (i) modelling paradigms themselves; (ii) perspectives of costs and benefits and (iii) time frame.

Methods: Papers that use modelling approaches for economic evaluations of drug and alcohol interventions were identified by carrying out searches of major databases.

Results: Thirty eight papers met the inclusion criteria. Overall, the cohort Markov models remain the most popular approach, followed by decision trees, Individual based model and System dynamics model (SD). Most of the papers adopted a long term time frame to reflect the long term costs and benefits of health interventions. However, it was fairly common among the reviewed papers to adopt a narrow perspective that only takes into account costs and benefits borne by the health care sector.
\end{abstract}

Conclusions: This review paper informs policy makers about the availability of modelling techniques that can be used to enhance the quality of economic evaluations for drug and alcohol treatment interventions.

Keywords: Substance abuse, Drug dependence, Alcohol dependence, Economic evaluation, Modelling

\section{Background}

\section{Economic evaluations and the role of modelling}

The economic, social and health consequences related to the consumption of drug and alcohol are a global concern with $0.8 \%$ of the global all-cause disability adjusted life years (DALYs) attributable to illicit drugs and alcohol [1]. Governments are faced with difficult decisions of how to best to allocate the limited available resources in order to maximise the value of spending (e.g. to achieve the highest net/social benefit). Modelling is frequently being applied as a method of depicting the complexity of decision making with respect to interventions and to

\footnotetext{
* Correspondence: v.hoang@unsw.edu.au

${ }^{1}$ Drug Policy Modelling Program, National Drug and Alcohol Research

Centre, University of New South Wales, Sydney, NSW 2031, Australia

Full list of author information is available at the end of the article
}

more accurately capture associated costs and benefits in economic evaluations.

Modelling can be broadly defined as the reproduction of events and possible consequences due to alternative policy options at the cohort or individual levels using mathematical and statistical framework. In the drug and alcohol area, models have been developed, for example, to mimic the heroin use and treatment profile of individuals over their life time, from which realistic outcomes can be calculated $[2,3]$. Despite modelling being widely applied for analysis of treatment for alcohol and drug problems, comprehension about which models are suitable to use for which research questions and how to interpret the results and caveats of the models remains limited. The primary aim of this paper, therefore, is to review the use of economic modelling in the evaluation of drug and alcohol treatment. Three modelling aspects 
are the focus of this review: (i) modelling approaches, (ii) perspectives of costs and benefits, and (iii) time frame (e.g. short or long-term time frame). Additionally, the concerns and relevance of each type of model will be discussed.

Decisions about the perspective of costs and benefits may have substantial impacts on the modelled outcomes due to the multiplicity of effects of interventions such as crime, transmission of infectious diseases, and other long-term consequences of drug and alcohol dependence. We adopt four distinct perspectives of costs and benefits according to common definitions in health economics as follows: "(i) individual patient - all consequences that accrue to the patient and all costs that are borne by the patient; (ii) health funder - for example, the costs that fall on a State health authority; (iii) health care sector - all costs that fall to the health sector, including, but not limited to, hospitals, specialists, general practitioners, ancillary services and community services (includes all health improvements or health-related quality-of-life improvements but does not include such things as informal carer costs, patient transport costs or time off work); and (iv) society - all costs and consequences that arise from the options no matter who pays or who receives benefits from them" [4]. In addition, different types of interventions may generate immediate or long-term effects. Therefore, for an economic evaluation to provide relevant policy advice, it should carefully account for the major costs and benefits and relevant time-frame associated with each intervention.

\section{Modelling classifications}

Economic evaluation is defined as a tool to compare the costs and benefits of alternative interventions, treatments or policy options. There is a suite of evaluation methods in health care research that may be used for different purposes. The search for information on efficiency often leads to an economic evaluation being conducted. Typically these have been cost-effectiveness (CEA) studies [5-7] or cost-utility (CUA) studies [8, 9]. While CEA and CUA studies can provide evidence to make the case for the investment in one treatment over another, the typically short-term time frame of the studies in the drug and alcohol field (e.g. 3 to 12 months) $[10,11]$ and the necessity to choose a single outcome means that they can fail to capture the full spectrum of costs and benefits. The single outcome, the narrow perspective taken by many studies and the exclusion of externalities such as costs of crime has led some to call for a greater focus on cost benefit analyses (CBA) when assessing interventions in the drug and alcohol field [12].

Cost-benefit analysis (CBA), with their outcomes valued in monetary terms, allows for the inclusion of a broader range of outcomes and externalities. It is, however, this inclusion in the CBA of a range of outcomes, and the necessity to value them in monetary terms that provides a number of challenges when conducting a CBA [13].

For those who are dependent on drug and alcohol there maybe movement in and out of treatment and prison, and the presence of co-morbid medical conditions such as anxiety disorders, depression, HIV/AIDs, Hepatitis C, and overdose [14-16]. Short-term economic evaluations conducted alongside RCTs are not usually sufficient to capture this multiplicity of outcomes. This problem has been long recognised when conducting economic evaluations of interventions for chronic illnesses. For example, in areas such as diabetes and heart disease there has been a shift to the use of economic modelling which can account for a multiplicity of outcomes and the long term and complex nature of the disease $[17,18]$.

The improvements in computer technology and development of new methods have made modelling a powerful tool to inform health policies. Through the synthesis of evidence from multiple datasets on the impact of interventions, behaviour changes of the individual and externalities, models can be constructed to compare the alternative interventions over a long timeframe. In general, modelling offers several advantages including: (i) extrapolating beyond the data observed in a trial; (ii) linking intermediate clinical endpoints to final outcomes; (iii) generalizing to other settings; (iv) synthesizing headto-head comparisons where relevant; and (v) informing decisions in the absence of hard data [19]. Broadly, a model includes four components: (i) the individuals/cohort under study; (ii) the states; (iii) the rules of transition between states over time; and (iii) the associated costs and benefits attached in each state. The complexity of a model depends on how the model components are defined. There exist several classification systems for health economic models. For example, Brennan et al. [20] proposes a classification based on whether a model is cohort or individual-based, discrete or continuous time-dependent and whether or not it allows for interactions between individuals. As with many classification systems, simplifications are often necessary to nudge the models into fixed categories, and many models may actually cross boundaries. Table 1 provides examples of common modelling paradigms classified according to two criteria proposed by Brennan et al. [20].

Decision tree models are the simplest and historically the most widely used form of models in health economics: all patient pathways are clearly laid out with their associated probabilities and outcomes (i.e. costs related to events, mortality rate, and the probability of committing crime) which are entered at the terminal node. The mean value of each decision in the tree is calculated by 
Table 1 Classification of modelling paradigms

\begin{tabular}{lll}
\hline & Cohort-based & Individual-based \\
\hline No interaction allowed & Decision treeCohort Markov model & Individual-based microsimulation/Markov microsimulation \\
Interaction allowed & System dynamics model & Agent based modelDiscrete event simulation
\end{tabular}

Adapted from Brennan et al. [21]

summing probabilities associated with each outcome. These models are usually used when the time frame is short, the process is not complicated, reoccurring events are not important, there is little heterogeneity between individuals in terms of outcomes and there is no interaction between individuals [20-22].

Cohort Markov models are suited to circumstances where the order of events is important, events may repeat, and the events may occur over a longer period of time. However, in addition to not allowing interaction between individuals in the model, cohort Markov models do not allow the probability of transitioning to another state in the model to depend upon the time spent in that state or previous history [20]. System dynamic models are also cohort-based models but allow interaction among entities by modelling the rate of change of the system to be dependent on the system itself (i.e. feedback loops).

Individual-based models can simulate a richer heterogeneity of individual trajectories, allowing for more realistic and complex patterns of disease evolution to emerge. For example, Individual-based microsimulation/ Markov microsimulation can simulate the life-time trajectories of participants and record participants' history. The transitions across different states may be conditional on previous events/history that participants have gone through. Agent-based models (ABM) include autonomous agents whose behaviour depends upon their past and current internal states, as well as states from other agents and their environment. Discrete event simulations (DES) allow for both modelling individual trajectories and interaction between entities in the system [20].

It is apparent, even from these brief descriptions, that different models are appropriate for different purposes. For example, models which are being used to generate evidence surrounding communicable disease will need to account for interaction between agents (i.e. ABM, DES) whereas a brief intervention for low risk alcohol consumption may only require a decision tree model. In order for the results to be meaningful, the choice of a modelling paradigm needs to reflect the problem being addressed and its context.

\section{Methods}

Papers that use modelling approaches for economic evaluations of drug and alcohol health interventions were identified by carrying out searches on major databases including: SCOPUS, Medline, CINAHL, PyscInfo, EconLit and ISI Web of Sciences. The search terms were: (drug dependence treatment OR substance abuse
OR heroin OR methadone OR buprenorphine OR cocaine OR Methamphetamine OR Cannabis OR opioid dependence OR alcohol OR dependence) AND (economic evaluation OR cost-effectiveness OR cost-utility OR costbenefit) AND (model* OR simulation). The inclusion criteria were: (i) economic evaluation studies of drug and alcohol health interventions; (ii) incorporating a modelling approach; (iii) peer-reviewed articles in academic journals published in the period from database inception to September 2015 in the English language. The exclusion criteria, therefore, were papers that were not economic evaluation, did not use modelling or were not about drug and alcohol health interventions. Accordingly, there were drug and alcohol modelling studies that were not met inclusion criteria such as: the simulation of the life course of cannabis use [23]; the simulation of Australian drug markets [24]; the examination of the impact of ecstasy pill-testing on the prevalence of harms [25] and exploring policy options and risks for alcohol consumption [26, 27].

Ethics for this study was not required because this study involved the analysis of published literature.

The initial search of databases yielded 1405 papers; 751 duplicate and unrelated papers were eliminated by checking titles, resulting in 654 papers. Next, each abstract was reviewed during which 81 papers were selected. The final step was to conduct in-depth content review of the selected papers with respect to modelling approach and economic evaluation framework. Those papers that were not a cost-benefit, a cost-effectiveness, or a cost-utility analysis of a health intervention for drug or alcohol problems, and did not use modelling methods were excluded ( $n=43$ exclusions). Finally, 38 papers were included in the review (see Fig. 1 and Additional file 1). During all screening steps, a set of papers was allocated to two authors so that each paper was screened independently twice. Any discrepancy in the selection of papers was discussed between the authors to reach a mutual agreement.

\section{Results}

Tables 2 and 3 present a summary of classifications of reviewed papers in regarding to: modelling approaches, perspectives on costs and benefits and time frame.

\section{Modelling approaches}

In terms of types of model, decision trees were used in 11 papers (29\%), cohort-based Markov modelling in 20 papers (52\%), system dynamics in 3 papers $(8 \%)$, individual based model in 2 papers (5\%) and another model 


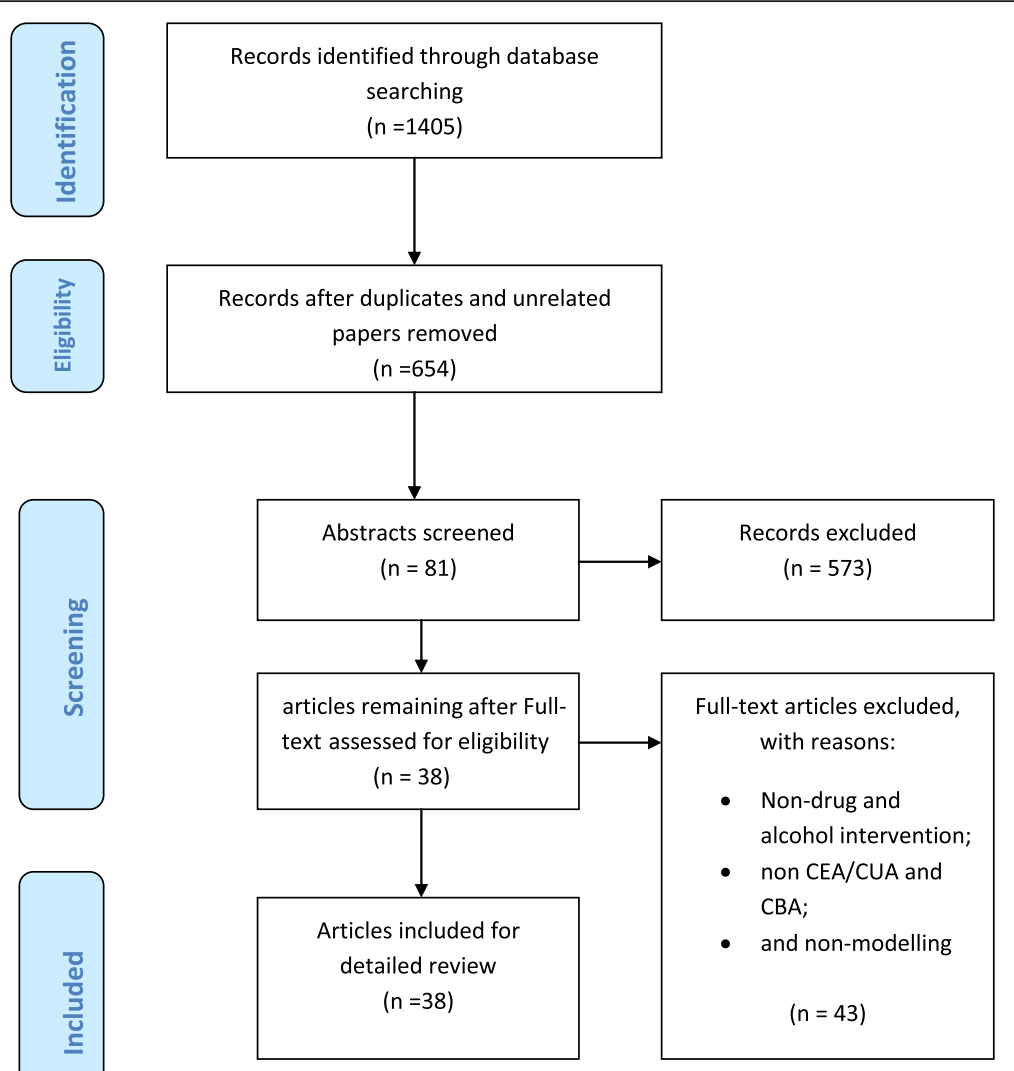

Fig. 1 Flow chart of literature search

Table 2 Classifications of reviewed papers

\begin{tabular}{|c|c|c|c|c|c|c|c|}
\hline Models used & & $\begin{array}{l}\text { Decision Tree } \\
(n=11)\end{array}$ & $\begin{array}{l}\text { Cohort Markov } \\
(n=20)\end{array}$ & $\begin{array}{l}\text { Individual-based } \\
\text { microsimulation } \\
\text { Models }(n=2) \\
\end{array}$ & $\begin{array}{l}\text { System Dynamics } \\
\text { Model }(n=3)\end{array}$ & $\begin{array}{l}\text { Other Models } \\
(n=2)\end{array}$ & $\begin{array}{l}\text { Total } \\
(n=38)\end{array}$ \\
\hline \multirow[t]{4}{*}{ Design } & & - Aggregate & - Aggregate & - Individual & - Aggregate & - Aggregate & \\
\hline & & $\begin{array}{l}\text { - No accounting for } \\
\text { heterogeneity }\end{array}$ & $\begin{array}{l}\text { - Limited } \\
\text { heterogeneity } \\
\text { by using states }\end{array}$ & $\begin{array}{l}\text { - Flexibility to include } \\
\text { heterogeneity by } \\
\text { using transition } \\
\text { probability function }\end{array}$ & $\begin{array}{l}\text { - Limited } \\
\text { heterogeneity by } \\
\text { using states }\end{array}$ & $\begin{array}{l}\text { - No } \\
\text { heterogeneity }\end{array}$ & \\
\hline & & - Untimed & - Timed & - Timed & - Timed & $\begin{array}{l}\text { - Untimed: only } \\
\text { before and after } \\
\text { intervention }\end{array}$ & \\
\hline & & - No history & - No history & - History & $\begin{array}{l}\text { - No history } \\
\text { - Allow interactions }\end{array}$ & - No history & \\
\hline \multirow[t]{2}{*}{$\begin{array}{l}\text { Perspective of } \\
\text { Costs and Benefits }\end{array}$} & $\begin{array}{l}\text { Health care } \\
\text { sector }\end{array}$ & 6 & 17 & 0 & 3 & 0 & 26 \\
\hline & Societal & 5 & 3 & 2 & 0 & 2 & 12 \\
\hline \multirow[t]{3}{*}{ Timeframe } & 0-1 year & 5 & 1 & 0 & 0 & 0 & 6 \\
\hline & $1-10$ years & 3 & 5 & 0 & 1 & 0 & 9 \\
\hline & $\begin{array}{l}10 \text { years to } \\
\text { life time }\end{array}$ & 3 & 14 & 2 & 2 & 2 & 23 \\
\hline
\end{tabular}


Table 3 Summary of papers and classification of modelling approaches, timeframe and perspectives in costs and benefits

\begin{tabular}{|c|c|c|c|c|c|c|c|}
\hline Authors/year of study and summary & Country & $\begin{array}{l}\text { Year of } \\
\text { study }\end{array}$ & $\begin{array}{l}\text { Analytic } \\
\text { method }\end{array}$ & Study participants & $\begin{array}{l}\text { Modelling } \\
\text { approach }\end{array}$ & $\begin{array}{l}\text { Time } \\
\text { frame }\end{array}$ & $\begin{array}{l}\text { Perspective on } \\
\text { costs and benefits }\end{array}$ \\
\hline $\begin{array}{l}\text { Barbosa et al. (2010) [41] have used a cohort based } \\
\text { probabilistic lifetime Markov model where alcohol } \\
\text { consumption and drinking history are used for classifying } \\
\text { patients into } 4 \text { Markov states. One year cycle length } \\
\text { was used for the model. The main outcomes were QALYs } \\
\text { and lifetime costs. }\end{array}$ & The U.K & 2010 & CEA & $\begin{array}{l}\text { Males who are seeking alcohol } \\
\text { treatment }\end{array}$ & Markov & Life time & Health care sector \\
\hline $\begin{array}{l}\text { Barnett et al. (2001) [8] have developed a dynamic } \\
\text { compartmental model to estimate the effect of adding } \\
\text { buprenorphine maintenance therapy to the US healthcare } \\
\text { system. The model divides population into mutually } \\
\text { exclusive groups ("compartments") based on HIV status } \\
\text { and drug use status. Transitions between these } \\
\text { compartments were modelled as a system of non-linear } \\
\text { differential equations. Current healthcare costs and } \\
\text { outcomes with the adoption of buprenorphine under } \\
\text { different scenarios were compared. }\end{array}$ & The U.S & 2001 & CEA & $\begin{array}{l}\text { Current population of methadone } \\
\text { treatment participants in the U.S } \\
\text { health care system }\end{array}$ & Markov & 10 years & Health care sector \\
\hline $\begin{array}{l}\text { Coffin and Sullivan (2013) [42] employs integrated cohort } \\
\text { based Markov and decision analytic model to assess } \\
\text { cost-effectiveness of distributing naloxone to heroin users } \\
\text { in the U.S over lifetime. In the model, heroin users enter the } \\
\text { model in 'Heroin use' state and can make transitions to } \\
\text { 'discontinue and relapse', 'overdose', or 'death by other } \\
\text { reasons' state. The 'overdose' state triggers a decision tree } \\
\text { based model based on naloxone distribution to assess } \\
\text { whether an individual will survive or die after overdose. }\end{array}$ & The U.S & 2013 & CEA & $\begin{array}{l}\text { Hypothetical 21-year-old novice U.S. } \\
\text { heroin user }\end{array}$ & Markov & Life time & Societal \\
\hline $\begin{array}{l}\text { Downs and Klein (1995) [28] developed cost-effectiveness } \\
\text { model based on decision trees for adolescent population } \\
\text { (15-19 years). The intervention is screening visits for alcohol } \\
\text { abuse and unsafe sexual activity. }\end{array}$ & The U.S & 1995 & CEA & Adolescents aged 15 to 19 years & Decision trees & 5 years & Societal \\
\hline $\begin{array}{l}\text { Magnus et al. (2012) [39] modelled economic and health } \\
\text { gains on the basis of an absolute change in alcohol } \\
\text { consumption. They modelled population simulation model } \\
\text { to determine lifetime benefits of a reduction in per capita } \\
\text { alcohol consumption from } 2008 \text { Australian adult cohort } \\
\text { (aged } \geq 15 \text { years). It considers workforce production gains } \\
\text { model, household production and leisure time model, and } \\
\text { health sector cost estimates for economic benefit evaluation. } \\
\text { This study aims to evaluate the benefits of reduction of } \\
\text { alcohol use thanks to a hypothetical intervention. }\end{array}$ & Australia & 2012 & N/A & The 2008 Australian population & Aggregate model & Lifetime & Societal \\
\hline $\begin{array}{l}\text { Navarro et al. (2011) [29] developed a decision tree based } \\
\text { model to assess outcomes and costs of GP-delivered }\end{array}$ & Australia & 2011 & CEA & Risky drinkers in 10 rural communities & Decision trees & 1 year & Health \\
\hline
\end{tabular}

model to as

intervention for alcohol misuse. Nine difference scenafios

with incremental increase in screening, brief intervention, or

in the combination of screening and brief intervention were

in New South Wales, Australia 
Table 3 Summary of papers and classification of modelling approaches, timeframe and perspectives in costs and benefits (Continued)

Purshouse et al. (2013) [30] developed a health economic model combining the healthcare resource requirements for alcohol screening and brief intervention with an

epidemiological model of relationships between alcohol consumption and health harms.

Sheerin et al. (2004) [40] used Markov model to model cohorts of injecting drug users, changes in their health states and effects of methadone maintenance therapy and anti-viral therapy on morbidity and mortality.

Wammes et al. (2012) [59] used Asian epidemic model and resource needs model to evaluate the long term preventive impact of expanding methadone maintenance therapy in West Java. In this model, population is divided into 8

compartments and individuals move from one compartment

to another based on a transition probabilities.

Tran et al. (2012) [32] developed a simulated decision tree based model to represent HIV-positive drug user's transition to 4 health state within one year horizon. Each of the states had services cost and health outcomes which were used for cost and benefit assessments.

Zaric et al. (2000) [43] developed a dynamic compartmenta model to assess the effects of increased methadone

maintenance capacity on healthcare costs and survival

(QALYS) for HIV epidemic in a population aged 18-44years.

Population is divided into 9 subgroups based on risk group and HIV infection status. Size of each compartment over time is modelled with the help of set of non-linear differential equations.

Tariq et al. (2009) [44] used a RIVM model to conduct a CEA of screening and brief intervention for alcohol in primary care targeting at reisk drinkers; outcomes were ICER, costs and QALY.

van den Berg et al. (2008) [45] used chronic disease model (CDM) to estimate the cost effectiveness of an alcohol tax increase from a health care perspective in the Netherlands; the outcomes were QALYS and LYS and health care costs

Vickerman et al. (2012) [36] used a system of differential equations to examine the impact on Hepatitis $C$ of scaling up OST and needle syringe programs;

Nosyk et al. (2012) [46] used a semi Markov cohort model to assess the increemental cost effectiveness of methadone versus diacetylmorphine in a cohort who had multiple failures of OST ; used data from the North American Opiate Medication Initiative trial; Outcomes used were QALYs and social costs (treatment, HIV, crime, calculated an ICER)

Zaric and Brandeau (2001) [37] used an epidemic model to determine optimal allocation of HIV prevention funds. Three
England

2013

CEA

Risky drinkers who are screened

through GP's visits

New Zealand

2004

CEA

2012 CEA

Indonesia

Vietnam

2012

HIV-positive drug users

Decision tree

1 year

Health care sector

The U.S

44

Netherlands

2009

The

Netherlands

2008

The U.K

Canada

CEA
Injecting drug users (IDUs)

Injecting drug users (IDUs)

Markov

20 years Societal
Risky drinkers aged between 20 and Markov 65 who visit the GP yearly $(50 \%)$

Current Dutch population

Markov

Injecting Drug Users (IDUs)

Injective drug users (IDUs)

Markov 
Table 3 Summary of papers and classification of modelling approaches, timeframe and perspectives in costs and benefits (Continued)

types of programs NSP (1), methadone (2), and condoms (3).

Outcomes were QALYs gained; and the investment portfolio

that maximises the number of HIV cases averted

Mortimer and Segal (2005) [47] used a time dependent

state-transition model to compare complementary and

competing interventions for prevention or treatment of

alcohol misuse and dependence; compares usual care with

interventions. Assesses proportions of patients drinking

beyond specified threshold, at 6,12 months follow-up; costs;

cost utility; used QALY league tables

Palmer et al. (2000) [48] uses a Markov model to explore the long term clinical and economic outcomes of alcohol

maintenance with counselling or counselling plus

accamprosate. Discounted and non-discounted LE and life

time costs, incremental cost effectiveness; uses abstinence.

Zaric et al. (2000) [49] uses a dynamic compartmental model of HIV to assess the cost effectiveness of MMT as a method of preventing HIV infection; the outcomes of the model are discounted LYS and QALYs and discounted health care and treatment costs

Adi et al. (2007) [31] investigates the clinical effectiveness and cost effectiveness of naltrexone for relapse prevention

in detoxified opioid dependent persons compared to

psychosocial support.

Barnett (1999) [50] examined cost effectiveness of methadone compared to standard care among cohort of 25 years old

heroin users in the U.S.

Bayoumi (2008) [51] examined cost effectiveness of medically supervised injecting centre; compared situation with

supervised injecting centre to no injecting centre but with needle syringe programs.

Alistar et al. (2011) [60] have developed a dynamic compartment Ukraine model of a population of IDUs on methadone substitution

therapy, IDUs injecting opiates and non-IDUs in order to

evaluate the effectiveness and cost effectiveness of

expanding methadone substitution therapy to IDUs,

increasing access to ART, or both. The outcome measures are the cost-effectiveness and QALYs.

Kapoor et al. (2009) [52] examine cost-effectiveness of various screening strategies for unhealthy alcohol use with \% Carbohydrate Deficient Transferrin using a Markov model.

Schackman et al. (2015) [62] evaluate the cost-effectiveness of long-term office-based buprenorphine/naloxone treatmen for clinically stable opioid-dependent patients compared to no treatment.

Australia

The U.S

The U.K

The U.S
Resource

allocation

framework

2005 CEA

Problem alcohol drinkers

Markov

Life time Health care sector

Germany

Problem alcohol drinkers

Markov

Life time Health care sector

2000

Injective drug users (IDUs)

Markov

10 years Health care secto

Injective drug users (IDUs)

Decision trees

1 year

Societal

1999

Injective drug users (IDUs)

Markov

Life time Health care sector

Injection drug users and persons infected with HIV and hepatitis virus

A population of non-IDUs, IDUs who inject opiates, and IDUs in MMT, adding an oral PrEP program (tenofovir/emtricitabine, $49 \%$ susceptibility reduction) for uninfected IDUs

Adult men and women (ages 18 to 100 years) in primary care

Cohort of clinically stable opioiddependent individuals who have already completed 6 months of office-based buprenorphine/naloxone treatment 
Table 3 Summary of papers and classification of modelling approaches, timeframe and perspectives in costs and benefits (Continued)

Tran et al (2012) [33] analyse the cost-effectiveness and budget impact of the methadone maintenance treatment

(MMT) programme in HIV prevention and treatment among

injection drug users (DUs) in Vietnam.

Zarkin (2012) [3] builds a Discrete event simulation to estimate the net societal benefits of diverting eligible poisoners to community based treatment in the U.S.

Zarkin et al. (2005) [2] estimate net societal benefits of providing methadone treatment in the U.S using Monte Carlo simulation model.

Rydell et al. (1994) [61] presents a model that estimates the relative cost-effectiveness of four cocaine-control programs: three "supply control" programs (source-country control,

interdiction, and domestic enforcement) and a "demand control" program (treating heavy users)

Cartwright (2000) [34] estimates the benefits of reduced cocaine consumption in terms of reduced societal costs resulting from the introduction of a medication for cocaine dependence with a small incremental treatment effect

Ciketic et al. (2015) [53] evaluates the cost-effectiveness of counselling as a treatment option for illicit MA use compared with no treatment option.

Alistar et al. (2014) [54] estimated the effectiveness and cost effectiveness of strategies for using oral PrEP in various combinations with methadone maintenance treatment (MMT) and antiretroviral treatment (ART) in Ukraine, a representative case for mixed HIV epidemics.

Angus et al. (2014) [55] adapt the Sheffield Alcohol Polic Model to evaluate a programme of screening and brief interventions (SBI) in Italy. Results are reported as Incremental Cost-Effectiveness Ratios (ICERs) of SBI programmes versus a 'do-nothing' scenario.

Jackson et al. (2015) [56] estimate the cost-effectiveness of injectable extended release naltrexone (XR-NTX) compared

to methadone maintenance and buprenorphine maintenance treatment (MMT and BMT respectively) for adult males

enrolled in treatment for opioid dependence in the United

States from the perspective of state-level addiction treatment payers.

Laramee et al (2014) [57] investigate whether nalmefene combined with psychosocial support is cost-effective compared with psychosocial support alone for reducing alcohol consumption in alcohol-dependent patients with high/very high drinking risk levels (DRLs) as defined by the $\mathrm{WHO}$, and to evaluate the public health benefit of reducing

harmful alcohol-attributable diseases, injuries and deaths.

Vietnam

2012

CEA

injection drug users (DUs)

Decision trees

1 year

Health-care secto

The U.S

The U.S

The U.S

1996

The U.S

2000

Australia

Ukraine

2014 CEA

Italy

2014

Adult males enrolled in treatment for Markov opioid

General population who visit GPs
A cohort of individuals who are incarcerated in the state prison system in the United States

The general population aged 18-60 (a percentage is heroin users)

The market includes the supply and demand of cocaine

Heavy cocaine users

Individuals recruited into

Methamphetamine Treatment Evaluation Study (MATES)

A population of non-IDUs, IDUs who Markov inject opiates, and IDUs in MMT

adding an oral PrEP program

(tenofovir/emtricitabine, $49 \%$

susceptibility reduction) for

uninfected IDUs.

Decision trees

30 years Societa 
Table 3 Summary of papers and classification of modelling approaches, timeframe and perspectives in costs and benefits (Continued)

\begin{tabular}{|c|c|c|c|c|c|c|c|}
\hline $\begin{array}{l}\text { Schackman et al (2015) [62] evaluate the cost-effectiveness of } \\
\text { rapid hepatitis C virus (HCV) and simultaneous HCV/HIV } \\
\text { antibody testing in substance abuse treatment programs. }\end{array}$ & The U.S & 2014 & CEA & $\begin{array}{l}\text { Opioid users in substance abuse } \\
\text { treatment programs }\end{array}$ & Decision trees & Life time & Health care sector \\
\hline $\begin{array}{l}\text { Thanh et al (2014) [63] used a decision analytic modeling } \\
\text { technique to estimate the incremental cost-effectiveness } \\
\text { ratio and the net monetary benefit of the Parent-Child } \\
\text { Assistance Program (P-CAP) within the Alberta Fetal Alcohol } \\
\text { Spectrum Disorder Service Networks in Canada. }\end{array}$ & Canada & 2015 & CEA & $\begin{array}{l}\text { Women who abuse substances } \\
\text { (e.g. alcohol and/or drugs) and are } \\
\text { pregnant }\end{array}$ & Decision trees & 3 years & Health care sector \\
\hline $\begin{array}{l}\text { Braithwaite et al (2014) [58] estimate the portion of HIV } \\
\text { infections attributable to unhealthy alcohol use and to } \\
\text { evaluate the impact of hypothetical interventions directed at } \\
\text { unhealthy alcohol use on HIV infections and deaths. }\end{array}$ & Kenya & 2014 & CEA & The Kenyan population & System dynamics & 20 years & Health care sector \\
\hline
\end{tabular}


type in 2 papers (5\%). The majority of studies were conducted in the US $(n=15)$, the U.K $(n=5)$, Australia $(n=4)$, Canada $(n=3)$ and the remainder were from a range of countries. Almost all of the studies used cost-effectiveness analysis (CEA) as the analytical method $(n=32)$, two studies used costbenefit analysis (CBA) and the rest used other analytical methods.

\section{Decision trees}

Eleven papers were found to have used a decision tree modelling approach. The topics ranged from short-term interventions for alcohol problem such as screening for alcohol users [28-30]; to relapse prevention for opioid use [31]; cost-effectiveness of methadone maintenance treatment (MMT) $[32,33]$ and providing medications to reduce cocaine dependence [34]. One paper, Navarro et al. [29] developed a decision tree model to assess outcomes and costs of a GP-delivered intervention for alcohol misuse for different scenarios (increase in screening; brief intervention; or a combination of screening and brief intervention and current practice). This paper, while providing an assessment of cost-effectiveness of different alternative interventions at an aggregate level, it does not take into account the role of individual attributes such as age, use and treatment history among the participants to determine the outcomes: effectively it assumes that the effect is the same for all participants. In the area of Opioid Substitution Treatment (OST), Tran et al. [32] developed a simulated decision tree model to evaluate cost-effectiveness of providing methadone to HIV-positive drug users in three treatment options; patients: (i) did not receive any drug substitution therapy during antiretroviral therapy (ART); (ii) received ART and MMT in standalone clinics, as currently delivered in Vietnam; and (iii) patients received ART under the direct supervision of health workers together with methadone during daily clinic visits. This model evaluated the outcomes at one-year after entering treatment and assumed the same treatment effects for all participants regardless of treatment history or use history. While these assumptions simplify the analysis and point to the key results, again they demonstrate the limitations in capturing the long term value of MMT treatment as well as the differential treatment effects across participants.

\section{Cohort Markov Models}

The majority of the reviewed papers used the cohort Markov modelling approach $(n=20)$ and cover a diverse range of drug and alcohol topics. One example of the application of cohort Markov model was an evaluation of cost-effectiveness of long-term outpatient buprenorphinenaloxone treatment for opioid dependence in primary care [35]. The cohort was allocated across four health states including 'In Treatment Off Drugs', 'In Treatment On Drugs', 'Out of Treatment Off Drugs', and 'Out of Treatment On Drugs'. The participants were then transitioned across states based on fixed transition probabilities. The outcomes including costs of treatment and QALYs (a utility based quality adjusted life year measurement which takes the value of 1 if full health and 0 if death) were attached to the states over the course of 24 months. This model demonstrates the key evolutions of opioid dependence and treatment over time and sets a clear structure for economic evaluation of treatment but has several limitations that are common in cohort Markov models. The lack of personal attributes, opioid use history and treatment history when determining either transition probabilities or costs and benefits of treatment mean that the model may not reflect the heterogeneity of the heroin treatment population.

\section{System Dynamics Model}

System dynamics modelling is used to account for the interaction between individuals in the cohort. These models are commonly used for infectious diseases such as HIV/AIDs and hepatitis C. For example, system dynamics models have been used in assessing the role methadone treatment has in reducing risky behaviours and new infections, which further reduces the rate of HIV infection in the cohort (i.e. feedback loops). We found three papers that applied this approach. The first paper examines the effect of needle-syringe programmes and opiate substitution treatment on the reductions in hepatitis $C$ virus prevalence in the UK [36]. The rates of infection are modelled through a system of differential equations that depend on susceptible and chronic injecting drug users in various interventions. The second paper evaluates the optimal investment to maximize life years gained or HIV infections averted in a population of infected drug users and non-users [37]. The optimization problem was set up with the objective of maximizing life years gained or HIV infections averted taking into account the non-linear growth of HIV infections. Despite the complexity of such models (and their relevance for infectious disease) one of the limitations of the system dynamics model is that it ignores individual attributes and history thus effectively limiting the heterogeneity of the cohort. However, the system dynamics modelling has an ability to depict the interaction among entities, which is crucial to obtain valid outcomes in the treatment and prevention of infectious diseases.

\section{Individual Based Models}

We found only two papers that met the review inclusion criteria for individual based microsimulation model. The first estimated the costs and benefits of providing methadone treatments in the context of the 
U.S. Here, Zarkin et al. [2] built an individual-based microsimulation that simulated the life time trajectories of a cohort of heroin users beginning at age 18 and ending at age 60. The participants cycled in and out of five mutually exclusive states including: (i) not using drugs and not in treatment; (ii) using drugs and not in treatment; (iii) in treatment; (iv) in prison and not using drugs and (v) in prison and using drugs. The key individual attributes such as age, gender, current heroin use, heroin use history and treatment history were used to determine the transition probabilities. While providing valuable results, the lack of data necessitated several simplifying assumptions. These included the assumption that the only treatment available was opioid substitution treatment and the transition probabilities were assumed to depend on only a few key individual attributes and heroin use and treatment history [2].

A second paper by Zarkin et al. [3] used a Discrete event simulation to assess the lifetime costs and benefits of diverting substance-abusing offenders from state prison to community-based treatment based on the premise that those offenders who undertook treatment have decreased criminal recidivism rates translating to costs-savings to the criminal justice system and gains from increasing probability of re-entering the labour market of offenders. This model captured the key events in the offenders' lifetime such as substance abuse, criminal activity, employment and treatment history and the transition probabilities are dependent on age, gender, and race and treatment history.

\section{Other models}

There are other ad-hoc modelling approaches, which are designed and used by researchers to conduct economic evaluations in particular circumstances that do not fit in the mainstream modelling paradigms. Two such papers are briefly described. Rydell et al. [38] modelled demand in the cocaine market that depended on the number of users, initiation rate, escalating and de-escalating use rates; and supply in the cocaine market that depended on the production amount minus the seized amount. The model was used to estimate the impact of different interventions such as treatment or law enforcement on supply and demand, from which different cost-effectiveness indicators can be compared. Magnus et al. [39] estimated the economic benefits to health, productivity and leisure due to a reduction of alcohol consumption with reference to the Australian population in 2008. They collected data about health and employment corresponding to different levels of alcohol consumption, from which economic benefits at different levels of alcohol consumption were estimated. These models provide population based evaluation outcomes, but do not take into account the complexity of disease evaluation such as interaction, recurrent events and heterogeneity of study subjects.

\section{Perspectives on costs and benefits}

The majority of the reviewed papers adopted a health care sector perspective $(n=26)$. The remaining papers adopted a broader perspective of costs and benefits by incorporating the social costs such as the impact of crime and benefits of employment in the evaluations of treatments [2, 3]. In assessing treatment interventions for alcohol and drug problems, it is clear that the selected perspective will impact on which costs and benefits are chosen and might impact on the assessment of net benefits of treatments enormously.

\section{Time frame}

The use of modelling permits examination of a longer time frame for economic outcomes, superior to economic evaluations conducted on individual RCT's with foreshortened timeframes. When the process is protracted as is frequently the case with dependence on drugs or alcohol, and thus where the costs and benefits of treatment are cumulative a longer timeframe is more appropriate. For the policy maker, this means economic projections into the distant future (and beyond the electoral cycle). The time frames used in the selected papers vary: there are six papers used 0-1 years; nine papers used 1-10 years and 23 papers used 10 years to life time. The majority of papers used cohort Markov, ISM and System Dynamics adopted long-term timeframe (e.g. ten years and up to lifetime). Zarkin et al. [2] illustrated that the choice of timeframe significantly influences the results in their study about the benefits and costs of methadone treatment in the U.S. They found that the benefit-cost ratio of methadone treatment produced from the lifetime model $(37.72: 1)$ was far more than the benefit-cost ratio from a static model of a one-off intervention (4.86:1).

\section{Discussion and conclusions}

Economic evaluations that incorporate modelling play an important role in producing realistic evidence for policy making around health interventions for drug and alcohol problems. The complexity of including the resource implications across multiple domains, including behaviours such as initiating drug use, increasing use, cycling in and out of treatment or prison during which previous events may alter the drug users' future life trajectories and affect the success of future treatments makes modelling in this field particularly challenging.

The decision tree modelling approach is a relatively easy technique for comparing different health interventions across a wide range of topics; however, it is not an 
optimal method when the population or treatment effects are heterogeneous, or when there are recurrent events, or when the outcome of interest is long-term. To this end, decision tree modelling may not be sufficient for evaluating treatments for alcohol and drugs.

On the other hand, the cohort Markov modelling maybe sufficiently sophisticated in that it may be able to replicate many of the potential developments in a disease process through the use of a series of health states. Particularly, it can account for both the time dimension by moving participants over time (e.g. time steps) and the heterogeneity of cohort by allocating them into relevant (but limited) states (e.g. poor health vs good health; types of treatment; drug dependent vs. not drug dependent). Attaching associated costs and benefits to states enables economic evaluations of various health interventions to be conducted. Although permitting more complex analyses than decision tree models, the Markov model relies on some major simplifications. First, it does not take into account the memory of past events that may shape participants' future transitions. In other words, the transition probabilities from one state to other possible states are fixed at any time in the model regardless of individual histories. Thus, these models cannot account for the number or length of previous treatment episodes. Secondly, it is often not feasible to take into account the full spectrum of heterogeneity of the population of interest in a Markov model. Again, this is a limitation if it is expected that these factors will significantly impact on the outcomes or costs.

In the area of interventions for drug and alcohol problems, the individual based model is a powerful tool as it is possible to simulate multiple events such as initiating heroin use, becoming dependent, cycling in and out of various modalities of treatment, entering and exiting prison over a period of time. The shortcomings in the Markov model are addressed by (i) incorporating the past events and personal attributes in determining transition probabilities; (ii) simulating participants' life trajectories individually rather than allocating participants into compartments; (iii) being flexible in time steps permitting participants to transit to the next state at different time intervals; (iv) providing a long-term perspective on costs and benefits of a series of health interventions over the participants' life time. However, a challenge Individual-based model is that it requires a large number of parameters to characterise the complexity of transitions across states and at different times and dependence on individual attributes.

The selection of a modelling approach is likely subject to the costs and time of model building. When evidence is required quickly, a decision tree can provide an initial estimation. However, when the problem pertains to multiple domains such as scaling up OST treatment and with substantial budget allocation, more complex models such as ISM or DES would be preferable.

The choice of an appropriate modelling approach, time frame and perspective of costs and benefits for a study may change the modelling results and policy implications significantly. Despite there is ample literature about the guidelines to identify an appropriate modelling framework [20], there is not sufficient justification and discussion about the choice of modelling aspects to match the complexity of an intervention in the reviewed papers. It is not uncommon to see different modelling approaches, time frames and perspectives of costs and benefits were used to examine a similar type of research questions. For example, in regarding to economic evaluation of methadone treatment, the time frames ranged from 1 year [33] to life time [2]; various modelling approaches were used ranging from decision tree [32], cohort Markov [40] and individual-based microsimulation [2]; and both health care sector and societal perspectives of costs and benefits were used [2, 33]. The other study also concluded the same findings with this review, where the authors pointed out that there is no consistent measuring framework of costs and benefits regarding to alcohol treatment [41]. Therefore, there is a pressing need to improve the transparency in modelling practice by offering justification for the choice of modelling aspects. The characteristics and consequences of an intervention should be discussed and the choice of modelling aspects should be justified. The interested readers may consult literature about modelling guidelines in health economics to aid their decision [20-22].

A limitation of this review is that it only focused on three main aspects used in study that include modelling approach, time frame and perspectives of costs and benefits, but not the process of decision about the choices of modelling aspects. Although this review have critically appraised a number of papers with respect to the appropriateness of the above modelling aspects, future research should evaluate the characteristics, effects and consequences of the intervention and recommend suitable modelling approach, time frame and perspectives of costs and benefits. This will provide evidence about the difference between the ideal model and the chosen model in the research of drug and alcohol intervention.

\section{Additional file}

Additional file 1: References to the list of selected papers for review. (DOC $84 \mathrm{~kb}$ )

\section{Abbreviations}

ABM: agent-based models; ART: antiretroviral therapy; CEA: cost-effectiveness analysis; DES: discrete event simulations; MMT: methadone maintenance treatment; OST: opioid substitution treatment; QALYs: quality-adjusted life years; RCTs: randomised control trials; SD: system dynamics models. 


\section{Competing interests}

The authors declare that they have no competing interests.

\section{Authors' contributions}

$\mathrm{VPH}, \mathrm{MS}, \mathrm{NS}$ and AR participated in the study concept and design, analysing the literature, and drafting and revising the manuscript. PP and MF participated in the study concept and design and drafting and revising the manuscript. All authors read and approved the final manuscript.

\section{Acknowledgements}

This paper was supported by Project Grant number APP1042923 from National Medical and Health Research Council (NMHRC) of Australia. The funder has no knowledge about this study.

\section{Author details}

'Drug Policy Modelling Program, National Drug and Alcohol Research Centre, University of New South Wales, Sydney, NSW 2031, Australia. ${ }^{2}$ SMART Infrastructure Facility, University of Wollongong, Wollongong, NSW 2522, Australia. ${ }^{3}$ National Drug and Alcohol Research Centre, University of New South Wales, Sydney, NSW 2031, Australia.

\section{Received: 14 May 2015 Accepted: 29 March 2016}

\section{Published online: 13 April 2016}

\section{References}

1. Degenhardt L, Whiteford HA, Ferrari AJ, et al. Global burden of disease attributable to illicit drug use and dependence: findings from the Global Burden of Disease Study 2010. Lancet. 2013;382:1564-74.

2. Zarkin GA, Dunlap LJ, Hicks KA, et al. Benefits and costs of methadone treatment: results from a lifetime simulation model. Health Econ. 2005:14: 1133-50.

3. Zarkin GA, Cowell AJ, Hicks KA, et al. Lifetime benefits and costs of diverting substance-abusing offenders from state prison. Crime Delinquency. 2012; $X X(X): 1-22$.

4. NHMRC. How to compare the costs and benefits: evaluation of the economic evidence. Canberra: NHMRC; 2001

5. Harris AH, Gospodarevskaya E, Ritter AJ. A randomised trial of the cost effectiveness of buprenorphine as an alternative to methadone maintenance treatment for heroin dependence in a primary care setting Pharmacoeconomics. 2005:23:77-91.

6. Bell J, Shanahan $M$, Mutch $C$, et al. A randomized trial of effectiveness and cost-effectiveness of observed versus unobserved administration of buprenorphine-naloxone for heroin dependence. Addiction. 2007;102: 1899-907

7. Doran CM. Buprenorphine, buprenorphine/naloxone and methadone maintenance: a cost-effectiveness analysis. Expert Rev Pharmacoecon Outcomes Res. 2005:5:583-91.

8. Barnett PG, Zaric GS, Brandeau ML. The cost-effectiveness of buprenorphine maintenance therapy for opiate addiction in the United States. Addiction. 2001:96:1267-78.

9. Vanagas G, Padaiga Z, Bagdonas E. Cost-utility analysis of methadone maintenance treatment in Lithuania. Medicina (Kaunas). 2010;46:286-92.

10. Eisenberg K, Woodruff SI. Randomized controlled trial to evaluate screening and brief intervention for drug-using multiethnic emergency and trauma department patients. Addict Sci Clin Pract. 2013;8(1):8. doi:10.1186/1940-0640-8-8

11. Saitz R, Palfai TA, Cheng DM, et al. Screening and brief intervention for drug use in primary care: the ASPIRE randomized clinical trial. JAMA. 2014;312(5): 502-13.

12. Sindelar $\mathrm{L}$, Jofre-Bonet $M$, French MT, et al. Cost-effectiveness analysis of addiction treatment: paradoxes of multiple outcomes. Drug Alcohol Depend. 2004;73:41-50.

13. Shanahan M, Ritter A. Confronting the challenges of conducting a CBA of cannabis policies. Drugs. 2013;20:175-83.

14. Sareen J, Chartier M, Paulus MP, et al. Illicit drug use and anxiety disorders: findings from two community surveys. Psychiatry Res. 2006;142:11-7.

15. Degenhardt L, Larney $S$, Randall D, et al. Causes of death in a cohort treated for opioid dependence between 1985 and 2005. Addiction. 2014;109:90-9.

16. Darke S, Mills KL, Ross J, et al. Rates and correlates of mortality amongst heroin users: findings from the Australian Treatment
Outcome Study (ATOS), 2001-2009. Drug Alcohol Depend. 2011;115: $190-5$

17. Lamotte $M$, Annemans $L$, Lefever $A$, et al. $A$ health economic model to assess the long-term effects and cost-effectiveness of orlistat in obese type 2 diabetic patients. Diabetes Care. 2002;25:303-8.

18. Schroettner J, Lassnig A. Simulation model for cost estimation of integrated care concepts of heart failure patients. Health Econ Rev. 2013;3:26.

19. Buxton MJ, Drummond MF, Van Hout BA, et al. Modelling in economic evaluation: an unavoidable fact of life. Health Econ. 1997;6:217-27.

20. Brennan A, Chick SE, Davies R. A taxonomy of model structures for economic evaluation of health technologies. Health Econ. 2006;15:1295-310.

21. Barton $P$, Bryan S, Robinson S. Modelling in the economic evaluation of health care: selecting the appropriate approach. J Health Serv Res Policy. 2004:9:110-8

22. Gray A, Clarke PM, Wolstenholme P, Wordsworth S. Applied Methods of Cost-effectiveness Analysis in Healthcare (Handbooks in Health Economic Evaluation). Oxford: Oxford University Press; 2010.

23. Paddock SM, Kilmer B, Caulkins JP, et al. An epidemiological model for examining marijuana use over the life course. Epidemiol Res Int. 2012; 2012:12.

24. Perez P, Dray A, Ritter A, Dietze P, Moore T, Mazerolle L. SimDrug: tackling the complexity of illicit drug markets in Australia. In: Perez P, Batten D, editors. Complex Science for a Complex World. Exploring Human Ecosystems with Agents. Canberra: ANU E Press; 2006. p. $193-224$

25. Moore D, Dray A, Green R, et al. Extending drug ethno-epidemiology using agent-based modelling. Addiction. 2009;104:1991-7.

26. Meier PS, Purshouse R, Brennan A. Policy options for alcohol price regulation: the importance of modelling population heterogeneity. Addiction. 2010;105:383-93.

27. White IR, Altmann DR, Nanchahal K. Alcohol consumption and mortality: modelling risks for men and women at different ages. BMJ. 2002:325:191-94.

28. Downs SM, Klein JD. Clinical preventive services efficacy and adolescents' risky behaviors. Arch Pediatr Adolesc Med. 1995:149:374-9.

29. Navarro HJ, Shakeshaft A, Doran CM, et al. The potential cost-effectiveness of general practitioner delivered brief intervention for alcohol misuse: evidence from rural Australia. Addict Behav. 2011;36:1191-8.

30. Purshouse RC, Brennan A, Rafia R, et al. Modelling the cost-effectiveness of alcohol screening and brief interventions in primary care in England. Alcohol Alcohol. 2013;48:180-88.

31. Adi $Y$, Juarez-Garcia A, Wang D, et al. Oral naltrexone as a treatment for relapse prevention in formerly opioid-dependent drug users: a systematic review and economic evaluation. Health Technol Assess. 2007;11:iii-iv, 1-85.

32. Tran BX, Ohinmaa A, Duong AT, et al. Cost-effectiveness of integrating methadone maintenance and antiretroviral treatment for HIV-positive drug users in Vietnam's injection-driven HIV epidemics. Drug Alcohol Depend. 2012;125:260-6

33. Tran BX, Ohinmaa A, Duong AT, et al. The cost-effectiveness and budget impact of Vietnam's methadone maintenance treatment programme in HIV prevention and treatment among injection drug users. Glob Public Health 2012;7:1080-94

34. Cartwright WS. Cocaine medications, cocaine consumption and societal costs. Pharmacoeconomics. 2000;18:405-13.

35. Schackman BR, Leff JA, Polsky D, Moore BA, et al. Cost-effectiveness of longterm outpatient buprenorphine-naloxone treatment for opioid dependence in primary care. J Gen Intern Med. 2012;27(6):669-76.

36. Vickerman P, Martin N, Turner K, et al. Can needle and syringe programmes and opiate substitution therapy achieve substantial reductions in hepatitis $C$ virus prevalence? Model projections for different epidemic settings. Addiction. 2012;107:1984-95

37. Zaric GS, Brandeau ML. Optimal investment in a portfolio of HIV prevention programs. Med Decis Making. 2001;21:391-408.

38. Rydell CP, Everingham SS. Controlling cocaine: Supply versus demand programs. Santa Monica, CA: RAND Corp; 1994. http://www.rand.org/pubs/ monograph reports/MR331.html.

39. Magnus A, Cadilhac D, Sheppard L, et al. The economic gains of achieving reduced alcohol consumption targets for Australia. Am J Public Health. 2012;102:1313-19. 
40. Sheerin IG, Green FT, Sellman JD. What is the cost-effectiveness of hepatitis $C$ treatment for injecting drug users on methadone maintenance in New Zealand? Drug Alcohol Rev. 2004;23:261-72.

41. Barbosa C, Godfrey C, Parrott S. Methodological assessment of economic evaluations of alcohol treatment: what is missing? Alcohol Alcohol. 2010; 45(1):53-63. doi:10.1093/alcalc/agp067.

42. Coffin PO, Sullivan SD. Cost-Effectiveness of Distributing Naloxone to Heroin Users for Lay Overdose Reversal. Ann Intern Med. 2013;158:1-U42.

43. Zaric GS, Barnett PG, Brandeau ML. HIV transmission and the costeffectiveness of methadone maintenance. Am J Public Health. 2000;90: 1100-11.

44. Tariq $L$, van den Berg M, Hoogenveen RT, et al. Cost-effectiveness of an opportunistic screening programme and brief intervention for excessive alcohol use in primary care. PLoS ONE [Electronic Resource]. 2009;4: e5696.

45. van den Berg M, van Baal PHM, Tariq $L$, et al. The cost-effectiveness of increasing alcohol taxes: a modelling study. BMC Med. 2008;6.

46. Nosyk B, Guh DP, Bansback NJ, et al. Cost-effectiveness of diacetylmorphine versus methadone for chronic opioid dependence refractory to treatment. CMAJ. 2012;184:E317-28.

47. Mortimer D, Segal L. Economic evaluation of interventions for problem drinking and alcohol dependence: Cost per QALY estimates. Alcohol Alcohol. 2005;40:549-55.

48. Palmer AJ, Neeser K, Weiss C, et al. The long-term cost-effectiveness of improving alcohol abstinence with adjuvant acamprosate. Alcohol Alcohol. 2000;35:478-92.

49. Zaric GS, Brandeau ML, Barnett PG. Methadone maintenance and HIV prevention: A cost-effectiveness analysis. Manage Sci. 2000;46:1013-31.

50. Barnett PG. The cost-effectiveness of methadone maintenance as a health care intervention. Addiction. 1999;94:479-88.

51. Bayoumi AM, Zaric GS. The cost-effectiveness of Vancouver's supervised injection facility. CMAJ. 2008;179:1143-51.

52. Kapoor A, Kraemer KL, Smith KJ, et al. Cost-effectiveness of screening for unhealthy alcohol use with \% carbohydrate deficient transferrin: results from a literature-based decision analytic computer model. Alcoholism. 2009; 33:1440-9.

53. Ciketic S, Hayatbakhsh R, McKetin R, et al. Cost-effectiveness of counselling as a treatment option for methamphetamine dependence. J Subst Use. 2015;20:239-46.

54. Alistar SS, Owens DK, Brandeau ML. Effectiveness and cost effectiveness of oral pre-exposure prophylaxis in a portfolio of prevention programs for injection drug users in mixed HIV epidemics. PLoS One. 2014;9, e86584.

55. Angus C, Scafato E, Ghirini S, et al. Cost-effectiveness of a programme of screening and brief interventions for alcohol in primary care in Italy. BMC Fam Pract. 2014;15:26.

56. Jackson H, Mandell K, Johnson K, et al. Cost-Effectiveness of Injectable Extended-Release Naltrexone Compared With Methadone Maintenance and Buprenorphine Maintenance Treatment for Opioid Dependence. Subst Abus. 2015;36:226-31.

57. Laramee P, Brodtkorb TH, Rahhali N, et al. The cost-effectiveness and public health benefit of nalmefene added to psychosocial support for the reduction of alcohol consumption in alcohol-dependent patients with high/very high drinking risk levels: a Markov model. BMJ Open. 2014;4, e005376.

58. Braithwaite RS, Nucifora KA, Kessler J, et al. How inexpensive does an alcohol intervention in Kenya need to be in order to deliver favorable value by reducing HIV-related morbidity and mortality? J Acquir Immune Defic Syndr. 2014;66:e54-8.

59. Wammes JJ, Siregar AY, Hidayat T, et al. Cost-effectiveness of methadone maintenance therapy as HIV prevention in an Indonesian high-prevalence setting: A mathematical modeling study. International Journal of Drug Policy. 2012;23:358-64

60. Alistar SS, Owens DK, Brandeau ML. Effectiveness and cost effectiveness of expanding harm reduction and antiretroviral therapy in a mixed HIV epidemic: a modeling analysis for Ukraine. PLoS Medicine / Public Library of Science. 2011;8:e1000423.
61. Rydell CP, \& Everingham SS. Controlling cocaine: Supply versus demand programs, Drug Policy Research Centre, RAND Corp. 1994.

62. Schackman BR, Leff JA, Barter DM, et al. Cost-effectiveness of rapid hepatitis $\mathrm{C}$ virus (HCV) testing and simultaneous rapid HCV and HIV testing in substance abuse treatment programs. Addiction (Abingdon, England). 2015;110:129-43.

63. Thanh NX, Jonsson E, Moffatt J, et al. An Economic Evaluation of the Parent-Child Assistance Program for Preventing Fetal Alcohol Spectrum Disorder in Alberta, Canada. Administration and Policy in Mental Health and Mental Health Services Research. 2014;42:10-18.

\section{Submit your next manuscript to BioMed Central and we will help you at every step:}

- We accept pre-submission inquiries

- Our selector tool helps you to find the most relevant journal

- We provide round the clock customer support

- Convenient online submission

- Thorough peer review

- Inclusion in PubMed and all major indexing services

- Maximum visibility for your research

Submit your manuscript at www.biomedcentral.com/submit
Biomed Central 